\title{
Shock-induced PDR in the Herbig-Haro object HH 2
}

\author{
B. Lefloch ${ }^{1}$, J. Cernicharo ${ }^{2}$, S. Cabrit ${ }^{3}$, and D. Cesarsky ${ }^{4}$ \\ ${ }^{1}$ Laboratoire d'Astrophysique de l'Observatoire de Grenoble, BP 53, 38041 Grenoble Cedex, France \\ e-mail: lefloch@obs.ujf-grenoble.fr \\ 2 Consejo Superior de Investigaciones Científicas, Instituto de Estructura de la Materia, Serrano 123, 28006 Madrid, Spain \\ 3 LERMA, Observatoire de Paris, UMR 8112, France \\ ${ }^{4}$ Max-Planck Institut für Extraterrestrische Physik, 85741 Garching, Germany
}

Received 25 May 2004 / Accepted 24 November 2004

\begin{abstract}
We report mid-infrared $(5-17 \mu \mathrm{m})$ and $\mathrm{SO}, \mathrm{CO},{ }^{13} \mathrm{CO}$ millimeter line observations of the protostellar jet $\mathrm{HH} 2$ and the parental molecular cloud. We have detected for the first time mid-infrared emission along a protostellar jet. We find that the outflowing gas extends much further away than the Herbig-Haro object HH 2, showing direct evidence that downstream gas has been accelerated by previous outflow events. These gas layers appear to have been detached from the parental cloud, as they are distributed around a cavity, probably dug by protostellar outflow(s). SO emission is detected in shocked gas regions associated with outflows. The UV field produced in the strong shock region $\mathrm{HH} 2 \mathrm{H}-\mathrm{A}$ has produced a low-excitation Photon-Dominated Region at the walls of the cavity, which is detected in the PAH emission bands and in the continuum between 5 and $17 \mu \mathrm{m}$. This continuum arises from very small grains transiently heated by a FUV field $\mathrm{G} \simeq 20 \mathrm{G}_{0}$, which probably formed from evaporation of dust grain mantles in shocks.
\end{abstract}

Key words. ISM: Herbig-Haro objects - ISM: individual objects: HH 1/2 - ISM: jets and outflows - ISM: dust, extinction stars: formation

\section{Introduction}

The Herbig-Haro system HH 1-2 is one of the best-studied star forming region. It attracted early attention because of the spectacular shock emission regions HH 1 and HH 2 that trace the interaction of the protostellar jet with the ambient cloud. This jet is characterized by rather high velocities in the ionic and atomic material (up to $480 \mathrm{~km} \mathrm{~s}^{-1}$ ) and large proper motions in the impact region, as measured with HST (Bally et al. 2002). The optical jet is associated with a molecular outflow, first mapped in CO by Moro-Martín et al. (1999). The jet makes a strong inclination angle with the line of sight $\approx 80^{\circ}$. The high-velocity, collimated component of the outflow (the "molecular jet") covers deprojected velocities of $15-80 \mathrm{~km} \mathrm{~s}^{-1}$. This system was first studied by Martin-Pintado \& Cernicharo (1987) who suggested that the spatial distribution of the molecular emission resulted from the interaction of the outflowing jet with the ambient molecular cloud, producing cavities and density enhancements along the walls of these cavities.

In the HH 2 region, the impact in the ambient gas results in shocks with a wide range of velocities: from $20-30 \mathrm{~km} \mathrm{~s}^{-1}$ in the rim, corresponding to the individual knots E-K (Lefloch et al. 2003) up to $180 \mathrm{~km} \mathrm{~s}^{-1}$ in the Mach disk region, which was identified as the optical knots $\mathrm{HH} 2 \mathrm{H}-2 \mathrm{~A}$. Observations in the UV (Boehm-Vitense et al. 1982; Raymond et al. 1997) and $\mathrm{X}$-ray (Pravdo et al. 2001) show extended emission from this shock region.
There has been a long debate as to whether such a highenergy field would have any noticeable impact on the local surroundings. Based on previous molecular line observations, the gas downstream of HH 2 appears to be in a quiescent state. However, Davis et al. (1990) report an enhanced abundance of $\mathrm{HCO}^{+}$in that region. Torelles (1992) obtained a similar result for $\mathrm{NH}_{3}$. A detailed study by Girart et al. (2002) revealed the peculiar chemical composition of the molecular emission peak in the region downstream of $\mathrm{HH} 2$ : some molecules were found largely overabundant $\left(\mathrm{CH}_{3} \mathrm{OH}, \mathrm{H}_{2} \mathrm{CO}, \mathrm{HCO}^{+}, \mathrm{SO}, \mathrm{SO}_{2}\right)$ and others, like CS and HCN, were underabundant. These features appeared to agree qualitatively with chemical models of UV-irradiated gas clumps (Wolfire \& Königl 1993; Viti \& Williams 1999), which predict large abundance enhancements in a wide variety of molecular species, from either purely gas phase reactions or the release from icy grain mantles. Timedependent modelling by Viti et al. (2003) could account for some of the molecular abundances measured by Girart et al. (2002), paying special attention to the $\mathrm{HCO}^{+}$emission. Other observational evidence of the impact of the UV field on the ambient gas around $\mathrm{HH} 2$ came from the observation of the FIR lines [CII] $158 \mu \mathrm{m}$ and [OI] at 63 and $145 \mu \mathrm{m}$ with ISO/LWS at $80^{\prime \prime}$ resolution by Molinari \& Noriega-Crespo (2002). They found that shock models cannot account for the line intensities measured and concluded that at least part of the emission must arise from a PDR associated with HH 2, but lack of angular 
resolution prevented any further characterization. Their modelling also made the assumption that the PDR is excited mainly by $2 \gamma$ UV photons.

On the other hand, it is well established that shocks can strongly alter the chemical composition in the entrained gas of protostellar outflows (see e.g. Bachiller \& Perez-Gutierrez 1997). Indeed, recent $\mathrm{HCO}^{+} J=1 \rightarrow 0$ interferometric observations by Dent et al. (2003) reveal a high-velocity component moving along $\mathrm{HH} 2$. The authors conclude that the observed $\mathrm{HCO}^{+}$abundance enhancement is consistent with shock chemistry in the turbulent mixing layer associated with the jet.

In this article, we reassess the nature of the molecular emission around $\mathrm{HH} 2$, from 5-17 $\mu \mathrm{m}$ spectro-imaging observations of the $\mathrm{HH} 2$ region obtained with the ISOCAM camera onboard ISO and complementary observations of millimeter transitions of $\mathrm{CO},{ }^{13} \mathrm{CO}$ and SO, at the IRAM $30 \mathrm{~m}$ telescope, paying special attention to the position studied by Girart et al. (2002), which we will refer to as the "molecular emission peak". Our observations show HH 2 to be even more complex than initially thought. We find that previous outflow episodes have accelerated gas ahead of $\mathrm{HH} 2$ and have dug a cavity in the parental cloud. Shocked gas is detected along the walls of the cavity. The mapping of SO emission shows that it is associated with the shock interaction of the outflowing gas with the parental cloud. The strong J-type shocks associated with HH 2H-A have induced the formation of a Photon-Dominated Region (PDR) at the inner wall of the cavity, which could be mapped and characterized in the mid-IR.

\section{Observations}

The $\mathrm{CO}$ and ${ }^{13} \mathrm{CO}$ millimeter line data were obtained at the IRAM 30m telescope and have been presented and discussed in Moro-Martín et al. (1999), hereafter MM99.

The SO transitions $3_{4}-2_{3}$ at $138.17864 \mathrm{GHz}$ and $2_{3}-1_{2}$ at 99.299883 GHz were observed with the IRAM 30m telescope in March 1993. The observing conditions were very good, with typical system temperatures of $180-200 \mathrm{~K}$ at $2 \mathrm{~mm}$. The angular resolution of the telescope is $17^{\prime \prime}$ and $24^{\prime \prime}$, respectively, at these frequencies. An autocorrelator with a spectral resolution of $20 \mathrm{kHz}$ was used as a spectrometer. The data was smoothed to obtain a kinematical resolution of $\approx 0.05 \mathrm{~km} \mathrm{~s}^{-1}$. The SO emission was mapped with a $15^{\prime \prime}$ sampling over a region of about $200^{\prime \prime}$ by $200^{\prime \prime}$ centered on the VLA 1 source. The flux is expressed in units of main-bream brightness temperature. The efficiency of the telescope was 0.65 and 0.55 at $99.299883 \mathrm{GHz}$ and $138.17864 \mathrm{GHz}$ respectively.

The mid-infrared observations were obtained with the ISO satellite (Kessler et al. 1996) and the ISOCAM instrument (Cesarsky et al. 1996). The low resolution spectra $(\lambda / \Delta \lambda=40)$ between 5 and $17 \mu \mathrm{m}$ were obtained in revolution 691 with the Circular Variable Filter (CVF) with a pixel scale of $6^{\prime \prime}$ and a total field of view of $3^{\prime}$ centered on the HH 2 object. We present the data reduced with the pipeline version OLP10. The size (HPFW) of the Point Spread Function (PSF) is $\approx 6^{\prime \prime}$ for a pixel scale of $6 "$.

Accurate astrometry (better than $2^{\prime \prime}$ ) was established using a second CVF map containing the optically visible
Cohen-Schwartz (CS) star, taken in revolution 873. Details are given in Cernicharo (2000) and Lefloch et al. (2003). The zodiacal light and a possible large-scale emission across the field were suppressed by subtracting a reference spectrum from the whole dataset. A spectrum of the residual emission, away from HH 2, is shown in panel A of Fig. 8c (the "empty field"). In addition to the CVF map we obtained an image of the flux integrated in the range 5.0-8.5 $\mu \mathrm{m}$, including the $\mathrm{H}_{2}$ pure rotational lines $\mathrm{S}(8)-\mathrm{S}(4)$ and the PAH bands at 6.2 and $7.7 \mu \mathrm{m}$. We extracted a map of the continuum emission in the range 13.9-15.5 $\mu \mathrm{m}$, outside the interval of emission of the [Ne 2], [Ne 3] and $\mathrm{H}_{2} \mathrm{~S}(2)$ lines. The data are presented in Fig. 8. Coordinates are offsets $(\operatorname{arcsec})$ relative to the position of VLA1: $\alpha_{2000}=05^{\mathrm{h}} 36^{\mathrm{m}} 22.6^{\mathrm{s}}, \delta_{2000}=-06^{\circ} 46^{\prime} 25^{\prime \prime}$.

The SNR of the data is not very high; we have averaged the signal over four fields typical of the region, which are drawn in Fig. 8: in the cloud (A), along the jet (D), and over the "ring" (B,C, see below). The interstellar extinction towards $\mathrm{HH} 2$ was estimated by Hartmann \& Raymond (1984), who measured typical reddenings $E(B-V)=0.11-0.44$. Based on the extinction curve of Rieke \& Lebofsky (1985), it appears that the flux dereddening corrections are negligible and we use uncorrected flux values in what follows.

\section{Entrained gas in the $\mathrm{HH} 2$ region}

Although the region has been extensively studied and several outflows identified (see e.g. Chernin \& Masson 1995; Correia et al. 1997) it is only recently (1999) that the molecular counterpart to the HH 1-2 jet was discovered (Moro-Martin et al. 1999). The confusion in the HH 2 region is indeed very high; because of the weakness of the outflow emission and a propagation close to the plane of the sky (about $10^{\circ}$, Noriega-Crespo et al. 1991), it is difficult to identify the different kinematical components in line spectra.

Several observations suggest that the medium in front of HH 2 has been accelerated in the past. Henney et al. (1994) showed that the medium in front of the counterjet of $\mathrm{HH} 2$ (HH 1) is moving at a considerable velocity, $200 \mathrm{~km} \mathrm{~s}^{-1}$. Ogura (1995) reported the presence of two giant bowshocks symetrically located at 30 arcmin from the protostellar core and aligned with $\mathrm{HH} 1-2$, ejected some $10^{4} \mathrm{yr}$ ago. These bowshocks were interpreted as the signature of previous ejections from the source powering the HH 1-2 jet. We have re-analyzed more thoroughly the ${ }^{12} \mathrm{CO}$ data presented by MM99 to search for any hint of outflow/ejections older than the $\mathrm{HH} 1-2$ jet mapped by them.

\subsection{A CO outflow ahead of $\mathrm{HH} 2$}

We show in Fig. 1 the CO $J=2 \rightarrow 1$ high-velocity gas emission in the HH 2 region, as mapped by MM99. The redshifted $\left(v_{\mathrm{lsr}}=12-16 \mathrm{~km} \mathrm{~s}^{-1}\right)$ outflow wing associated with the HH 2 jet extends between the driving source VLA 1 and the $\mathrm{HH}$ object (the $\mathrm{H}_{2}$ knots of shocked gas are marked by white stars in Fig. 1. Close inspection of the data reveals another $\mathrm{CO}$ component, well collimated, which propagates downstream of the red wing at blueshifted velocities $\left(v_{\mathrm{lsr}}=2-4 \mathrm{~km} \mathrm{~s}^{-1}\right)$. 

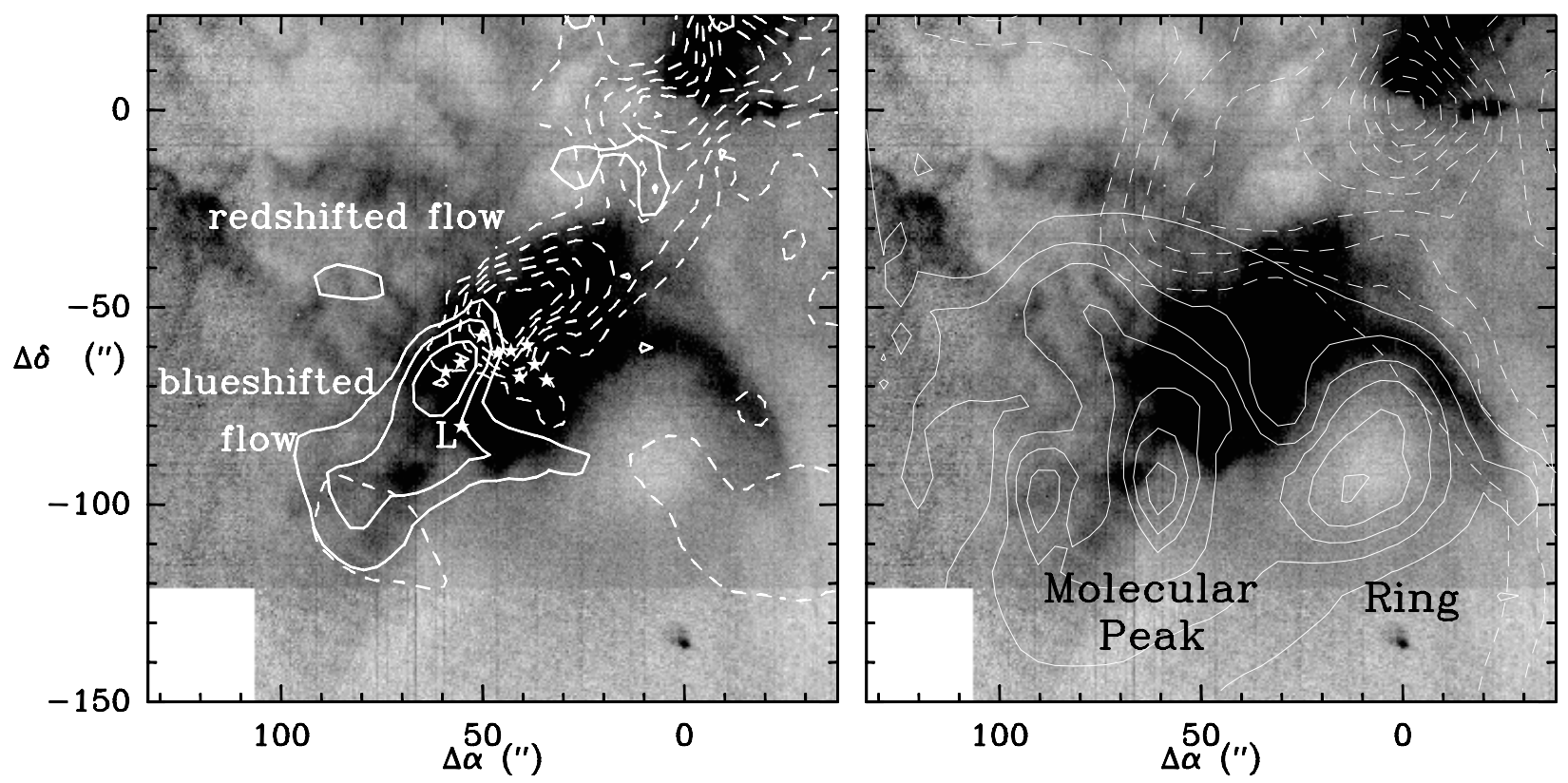

Fig. 1. (Left) Map of the high-velocity outflow emission in the ${ }^{12} \mathrm{CO}(2-1)$ line (thick black contours) superposed on the map of the ${ }^{13} \mathrm{CO}(2-1)$ emission (thick white contours) and a [SII] image of the region (greyscale; Reipurth et al. 1983). The image has been saturated to outline the ring and the weak emission downstream of HH2. The redshifted wing of the HH 2 outflow is drawn in dashed contours. Contours range from 4 to $20 \mathrm{~K} \mathrm{~km} \mathrm{~s}^{-1}$ by step of $2 \mathrm{~K} \mathrm{~km} \mathrm{~s}^{-1}$. The blueshifted wing is drawn in solid contours. First contour and contour interval are 1 and $0.5 \mathrm{~K} \mathrm{~km} \mathrm{~s}^{-1}$ respectively. The white stars mark the position of the $\mathrm{HH} 2 \mathrm{knots} \mathrm{A}$ to L. The position of the regions discussed in the text (the "ring" and the molecular emission peak downstream HH 2) are indicated. (Right) Map of the ${ }^{13} \mathrm{CO}(2-1)$ emission integrated between 5.5 and $6.75 \mathrm{~km} \mathrm{~s}^{-1}$ (thick white contours) and between 9.5 and $12 \mathrm{~km} \mathrm{~s}^{-1}$ (dashed) superposed on a [SII] image of the region (greyscale). Intensity contours range from 6 to $11 \mathrm{~K} \mathrm{~km} \mathrm{~s}^{-1}$.

A spectrum of the $\mathrm{CO}$ outflowing gas near the brightness peak at offset position $\left(60^{\prime \prime},-75^{\prime \prime}\right)$ is displayed in Fig. 2. The blueshifted wing overlaps very well the ambient gas layers, as traced by ${ }^{13} \mathrm{CO}$. The maximum of brightness in the blue component is detected in the region that coincides with the $\mathrm{HCO}^{+}$ high-velocity wing reported by Dent et al. (2003). This kinematical component extends from knots HH 2 K-E and propagates over $\approx 50^{\prime \prime}$, ahead of knot L (see Fig. 1). The optical [SII] emission of HH 2 reveals a faint jet that propagates ahead of HH 2 and coincides spatially with the CO blueshifted outflow (see left panel in Fig. 1). This jet is likely to be the driving source of the blueshifted molecular material.

A crude estimate of the parameters in the outflowing gas was made by assuming a kinetic temperature of $30 \mathrm{~K}$. As can be seen in Fig. 2, the CO spectra indicate antenna temperatures of $\simeq 20 \mathrm{~K}$, which is a lower limit to the intrinsic line brightness. The brightness temperature of the very optically thick $\mathrm{CO}$ lines provides a lower limit to the actual gas kinetic temperature, which is about $25 \mathrm{~K}$. An upper limit is provided by the mainbeam brightness temperature $\simeq 40 \mathrm{~K}$; we adopt an intermediate value of $30 \mathrm{~K}$ in this work. This value is characteristic of the ambient gas and may underestimate the actual temperature in the outflowing gas. It is higher than the $13 \mathrm{~K}$ estimated by Girart et al. (2002). These authors obtained this estimate indirectly, based on a multi-transition Monte-Carlo analysis of $\mathrm{HCO}^{+}$. We favor our approach, which relies directly on the observation of a "standard" tracer; excitation problems can be very severe for $\mathrm{HCO}^{+}$. The presence of a thermal gradient in the layers cannot be excluded, in which case the temperature
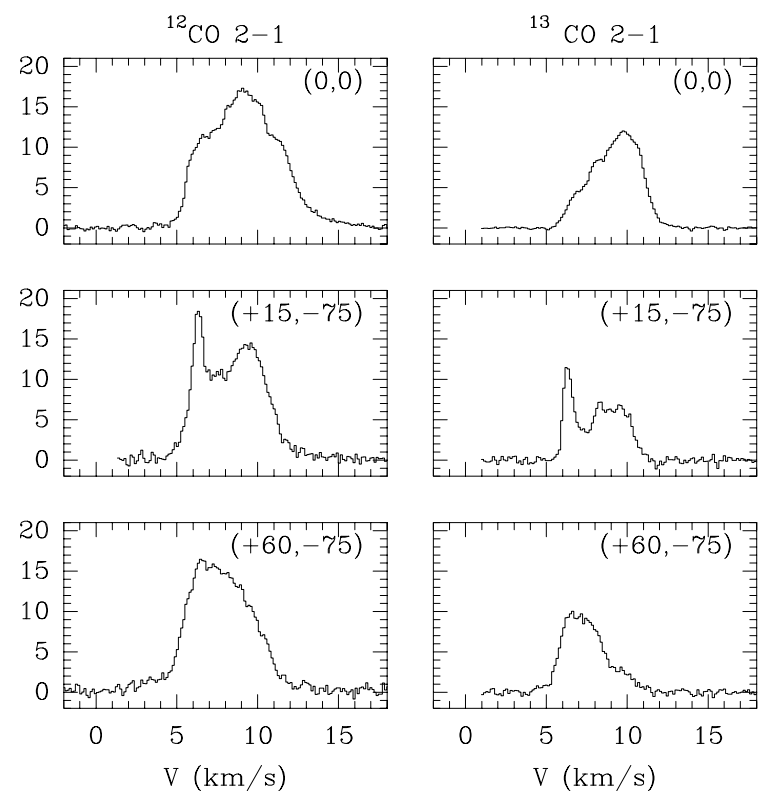

Fig. 2. Montage of ${ }^{12} \mathrm{CO}(2-1)$ and ${ }^{13} \mathrm{CO}(2-1)$ spectra at 3 positions: the protostellar core (top), the "ring" (middle), and the "molecular peak" downstream of HH 2 (bottom).

at the surface (traced by the low-excitation CO line) could be higher than in the inner denser regions probed by $\mathrm{HCO}^{+}$.

Integrating the wing emission in the range $2-4 \mathrm{~km} \mathrm{~s}^{-1}$, we find a mass of $0.015 M_{\odot}$ and a momentum of $0.1 M_{\odot} \mathrm{km} \mathrm{s}^{-1}$. These parameters compare well with those derived for the 


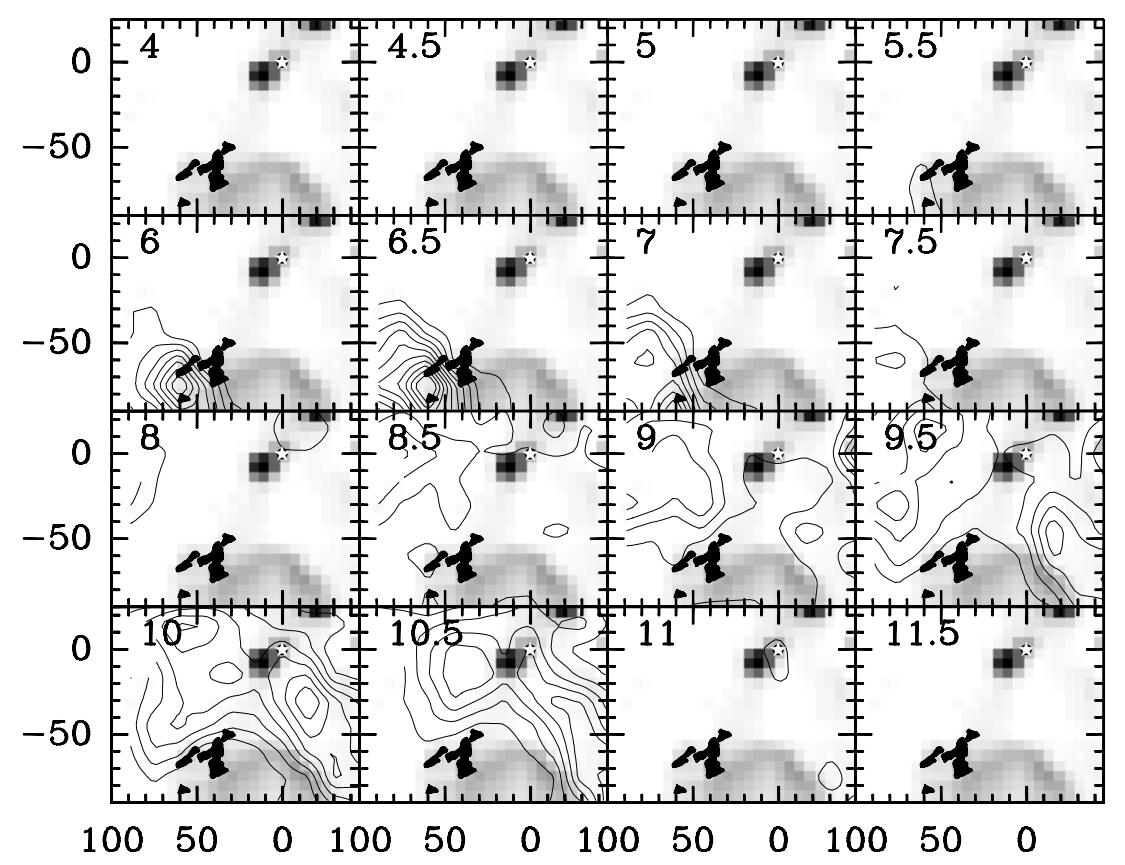

Fig. 3. Velocity-integrated intensity channel map of the SO $3_{4}-2_{3}$ emission in the HH 1-2 region (contours), superposed on the 5.0-8.5 $\mu \mathrm{m}$ emission map obtained with ISO. First contour and contour interval is $0.2 \mathrm{~K}$. Velocity is indicated in the top left corner of each panel. The position of VLA 1 is marked by a white star. The individual HH knots are drawn in black. Coordinates are in arcsec offset with respect to VLA 1.

high-velocity molecular outflow associated with the HH 2 jet. This blueshifted component appears relatively well collimated and is aligned with the redshifted Herbig-Haro jet HH 2, which suggests a common origin for both ejections. Both components slightly overlap at the position of the knots E-F-K, where the maximum of shocked $\mathrm{H}_{2}$ emission is detected in the mid-IR (Lefloch et al. 2003). The alignment and overlap is better seen in the higher-angular resolution observations of Dent et al. (2003). Though appealing, it is not clear if this change of orientation results from precession of the jet driving source (blue and red components would be tracing two different ejections) or from deflection upon impact on dense obstacle (the components are tracing one single jet). Both components have approximately the same (projected) size $\approx 50^{\prime \prime}=3.3 \times 10^{17} \mathrm{~cm}$, and could trace two ejections separated by $500 \mathrm{yr}$, assuming a typical ejection velocity of $200 \mathrm{~km} \mathrm{~s}^{-1}$. This blueshifted outflow and the optical jet downstream of $\mathrm{HH} 2$ provide direct evidence that material ahead of $\mathrm{HH} 2$ has already been accelerated by previous outflowing ejections.

\subsection{The high-density gas emission}

SO is a tracer whose emission is more highly contrasted than $\mathrm{CO}$ because its abundance can be greatly enhanced in shocks and in the high-temperature central protostellar regions (the "hot cores" of low- and high-mass protostars), where grain mantles are evaporated and S-bearing molecules released in the gas phase. SO is therefore especially suited to map the shocked gas of the outflow and its interaction with the ambient cloud. We detected extended SO emission over the entire surveyed region. We show in Fig. 3 a channel map of the velocityintegrated flux of the SO $3_{4}-23$ line. We found two maxima; the first one is located at position $\left(-75^{\prime \prime}, 12^{\prime \prime}\right)$, the second one lies very close to knot $\mathrm{HH} 2 \mathrm{~L}$ at position $\left(60^{\prime \prime},-75^{\prime \prime}\right)$, in very good agreement with the "molecular peak" position determined by Girart et al. (2002). At this position, SO lines are bright ( 4 K), more than twice as bright as in the protostellar core, where the $\mathrm{HH} 1$ jet is detected as a blue wing, and in the HH 2 outflow (see Fig. 4).

We concentrate on the region downstream $\mathrm{HH} 2$ in what follows. From the half-power contour, we estimate a transverse (deconvolved) size of $\approx 20^{\prime \prime}$, hence barely resolved by our observations. We show in Fig. 4 the profiles obtained in a 4-point cut in declination across the brightness peak at $\left(60^{\prime \prime},-75^{\prime \prime}\right)$. The line profiles peak at about $6.5 \mathrm{~km} \mathrm{~s}^{-1}$. However, they are characterized by several kinematical components, which vary between adjacent positions, separated by $15^{\prime \prime}$. At $\delta=-90^{\prime \prime}$, a secondary component is detected at $6.89 \mathrm{~km} \mathrm{~s}^{-1}$. The velocity channel map suggests that the $7 \mathrm{~km} \mathrm{~s}^{-1}$ component peaks at lower declination, near position $\left(50^{\prime \prime},-90^{\prime \prime}\right)$. Unfortunately, the data sampling is lower in that region $\left(30^{\prime \prime}\right)$ and does not allow us to resolve details in the structure of this feature. North of the peak $\left(\delta>-60^{\prime \prime}\right)$, a secondary component is detected at $10 \mathrm{~km} \mathrm{~s}^{-1}$. It is is rather weak, with $T_{\mathrm{mb}} \sim 1 \mathrm{~K}$, but it is unambiguously detected across the whole map. It is spatially associated with low-redshifted gas which extends from the VLA 1 protostellar core to $\mathrm{HH} 2$ and beyond. Overall, the gas traced by SO does not appear quiescent in the region downstream of $\mathrm{HH} 2$.

\subsubsection{Physical properties of the SO emission}

We have carried out an LVG analysis of the SO emission at a few positions close to the brightness peak region, considering one single gas component. We have neglected any thermal and/or density gradients in the emitting gas layer, despite the 

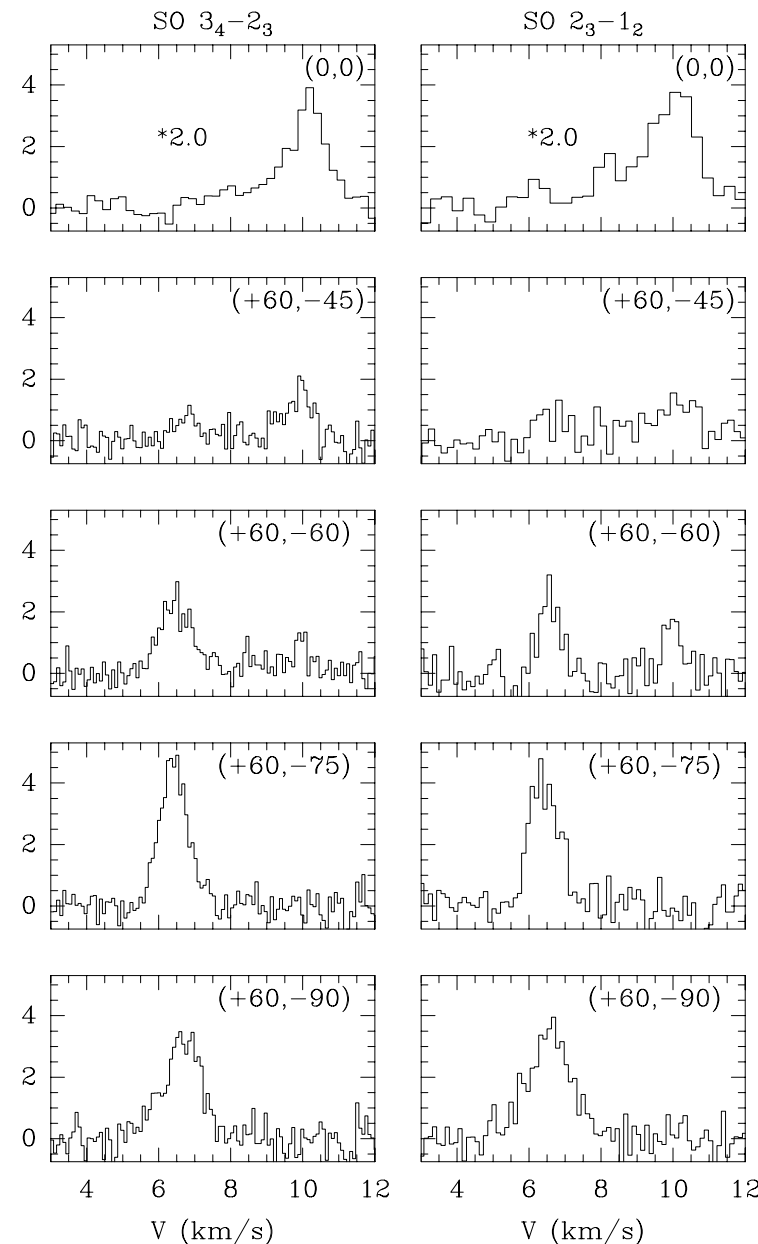

Fig. 4. Spectra of the SO $3_{4}-2_{3}$ and $2_{3}-1_{2}$ lines observed towards the molecular emission peak downstream HH 2 at offset position $\left(60^{\prime \prime}\right.$, $\left.-75^{\prime \prime}\right)$ and the protostellar source VLA 1. Flux is in main-beam brightness temperature. Line intensities have been multiplied by 2.0 for the VLA 1 spectra.

fact that the SO emission arises from a shocked gas layer, as we discuss below in Sect. 3.2.2. Taking into account such an effect would introduce an additional degree of freedom in the modelling, for which the present observational data set does not bring any constraints. We adopt a kinetic temperature of $30 \mathrm{~K}$, similar to that determined in the lower-density gas traced by $\mathrm{CO}$ (see above). Collisional rates for SO have been derived from those of CS- $\mathrm{H}_{2}$ computed by Green \& Chapman (1978); the procedure for ${ }^{3} \Sigma$ molecules is described by Fuente et al. (1990). We obtained very similar results using the more recent collisional rates computed by Green (1994) and extrapolated at temperatures lower than $50 \mathrm{~K}$.

At the peak, both lines are bright with main beam temperatures of $4.2 \mathrm{~K}$ and $4.7 \mathrm{~K}$ for the $2_{3}-1_{2}$ and $3_{4}-2_{3}$ transitions respectively. Linewidths are $\approx 1 \mathrm{~km} \mathrm{~s}^{-1}$. We find a solution for a column density $N(\mathrm{SO})=5.6 \times 10^{13} \mathrm{~cm}^{-2}$ and a gas density $n\left(\mathrm{H}_{2}\right)=5.5 \times 10^{5} \mathrm{~cm}^{-3}$. The lines are optically thin with opacities of 0.45 and 0.23 for the $3_{4}-2_{3}$ and $2{ }_{3}-1_{2}$ respectively. The LVG calculations predict intensities of $2.2 \mathrm{~K}$ for the $5_{6}-4_{5} 219.9494 \mathrm{GHz}$ and $0.91 \mathrm{~K}$ for the $6_{7}-5_{6} 261.8437 \mathrm{GHz}$, both transitions observed by

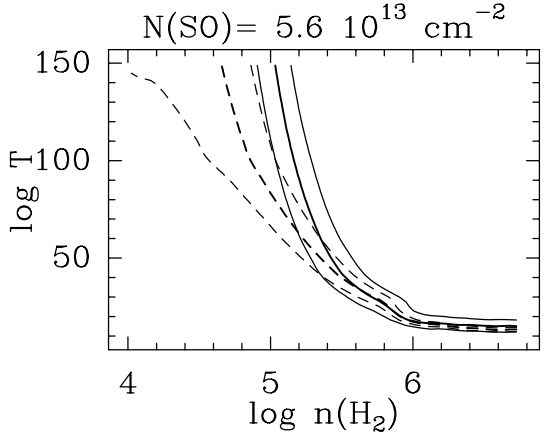

Fig. 5. Variations of the SO $3_{4}-2_{3} / 2_{3}-1_{2}$ (thick solid) and $6_{7}-5_{6} / 5_{6}-4_{5}$ (thick dashed) intensity line ratios with temperature and density at the molecular peak. The SO column density is taken to be $5.6 \times 10^{13} \mathrm{~cm}^{-2}$ (see text). Thin contours draw the values at 90 and 110 .

Girart et al. (2002) at the SO peak. This is in good agreement with the line brightness, once corrected for the beam dilution factor (respectively 0.257 and 0.322 ).

We note that a lower temperature, of the order of $15 \mathrm{~K}$ for the emitting gas, would require much higher densities, of the order of $n\left(\mathrm{H}_{2}\right)=5 \times 10^{6} \mathrm{~cm}^{-3}$, and $N(\mathrm{SO}) \simeq 6.5 \times 10^{13} \mathrm{~cm}^{-2}$. These densities are far too high and lead to a flux much larger than what is observed for the $56-45$ and $6{ }_{7}-5_{6}$ transitions (2.7 K and $1.3 \mathrm{~K}$ respectively). These results differ somewhat from the simple LTE analysis by Girart et al. (2002). Indeed, the excitation temperature ranges from $24 \mathrm{~K}$ for the $2_{3}-1_{2}$ transition down to $9 \mathrm{~K}$ for the $6_{7}-5_{6}$ transition, showing that these transitions are far from being thermalized.

Taking into account the uncertainties in the brightness determination, it appears that various sets of physical conditions can account for the observed lines. We have explored the range of solutions in the parameter space defined by the density and the temperature, adopting the SO column density determined above at the brightness peak (the "best solution"). We show in Fig. 5 the intensity line ratios $34-2_{3} / 2_{3}-1_{2}$ (thick solid line) and $6{ }_{7}-5_{6} / 5_{6}-4_{5}$ (thick dashed line). A good agreement is obtained for densities in the range $3-6 \times 10^{5} \mathrm{~cm}^{-3}$ and temperatures between 25 and $40 \mathrm{~K}$. Allowing a variation of $10 \%$ for each ratio, we find that the density has to be more than $10^{5} \mathrm{~cm}^{-3}$, and the temperature less than $100 \mathrm{~K}$. If the density $n\left(\mathrm{H}_{2}\right)$ is larger than $2.5 \times 10^{5} \mathrm{~cm}^{-3}$ i.e. the average gas density in the layer (see Sect. 4.1), the temperature is relatively well constrained, between $20 \mathrm{~K}$ and $60 \mathrm{~K}$. Observations of higher-excitation transitions of SO (and $\mathrm{CO}$ ) would help constrain the temperature.

Comparison of the relative intensities at the neighbouring positions (Fig. 4) shows that the $3_{4}-2_{3}$ intensities is lower than $2_{3}-1_{2}$, suggesting a change of excitation conditions, namely a lower density. An LVG analysis yields a column density $N(\mathrm{SO})=3.0-4.0 \times 10^{13} \mathrm{~cm}^{-2}$ and a density $n\left(\mathrm{H}_{2}\right) \simeq 2 \times$ $10^{5} \mathrm{~cm}^{-3}$. If the gas temperature were lower, typically $15 \mathrm{~K}$, the densities derived would be $n\left(\mathrm{H}_{2}\right) \simeq 6-7 \times 10^{5} \mathrm{~cm}^{-3}$, which is very unlikely for structures with a size of $10^{17} \mathrm{~cm}$ in the molecular cloud. The densities derived from our LVG approach are very similar to those quoted by Dent et al. (2003), 


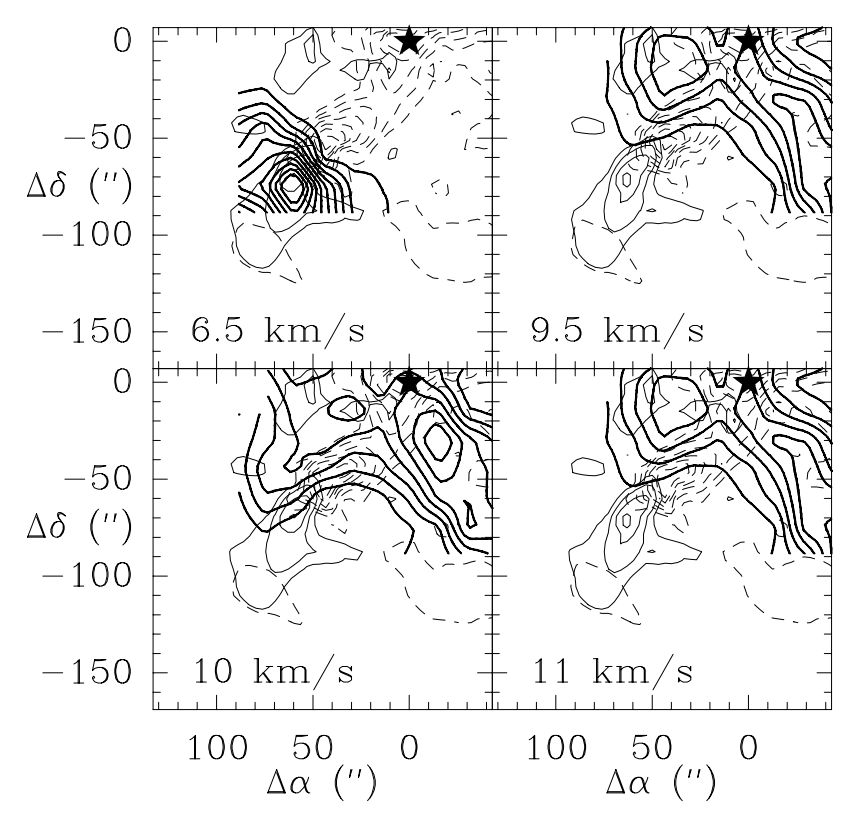

Fig. 6. Comparison of the emission distribution of the $\mathrm{SO}_{4}-2{ }_{3}$ (black contours) at various velocity intervals $\left(6.5,9.5,10,10.5 \mathrm{~km} \mathrm{~s}^{-1}\right)$ with the CO 2-1 high-velocity outflow. First contour and contour interval are $0.2 \mathrm{~K}$.

from millimeter dust continuum, and from modelling of the $\mathrm{HCO}^{+}$emission by Girart et al. (2002).

The secondary component at $10 \mathrm{~km} \mathrm{~s}^{-1}$ detected North of the SO peak $\left(\delta=-60^{\prime \prime}\right.$ and $\left.\delta=-45^{\prime \prime}\right)$ is characterized by highexcitation conditions with a ratio $3_{4}-2_{3} / 2_{3}-1_{2} \sim 1.5$. Such a high value is found only at the SO emission peak ahead of $\mathrm{HH} 2$ (it is less than 1 elsewhere in that area). Carrying out an LVG analysis of the position $\left(60^{\prime \prime},-45^{\prime \prime}\right)$ and adopting a kinetic temperature of $30 \mathrm{~K}$, we find a gas density $n\left(\mathrm{H}_{2}\right)=5 \times 10^{6} \mathrm{~cm}^{-3}$ and $N(\mathrm{SO})=2.0 \times 10^{13} \mathrm{~cm}^{-2}$. Again, the density estimate relies on the temperature adopted, but we stress that $30 \mathrm{~K}$ is probably a reasonable lower limit (lower temperatures require even higher densities, $\sim 10^{7} \mathrm{~cm}^{-3}$, irrealistically large for the largescale entrained gas of a molecular outflow). The emitting gas is therefore characterized by high densities, much larger than in the ambient parental cloud, and possibly warmer temperatures. Taking into account that this gas is moving into the ambient cloud, we conclude that at least part of the SO component is tracing gas shocked and/or accelerated by the HH 2 jet.

\subsubsection{Origin of $\mathrm{SO}$ emission}

How does the distribution of the SO-emitting gas relate to the $\mathrm{CO}$ outflowing gas detected in $\mathrm{HH} 2$ ? Figure 6 shows that SO traces the large-scale features associated with the accelerated gas detected in $\mathrm{CO}$ on each side of the protostellar core at $8.5 \mathrm{~km} \mathrm{~s}^{-1}$. In the protostellar core itself, the SO line profiles exhibit blue- and red-shifted wings, which are the signature of the HH 1-2 outflow. We did not find any evidence of emission along the jet down to HH 2; instead, we detect in the same velocity range emission that is spatially shifted with respect to the jet; such a shift could be an indication that the emission arises from the jet rim, i.e. the low-velocity shock region of the jet (see Lefloch et al. 2003). The gas column densities derived are indeed comparable to the values encountered in other young protostellar outflows, like L1157 (Bachiller \& Perez-Gutierrez 1997). Observations at higher-angular resolution would help clarify this point.

As discussed above, the high-velocity blushifted outflow propagates into the gas layers downstream of $\mathrm{HH} 2$, where SO is detected at velocities equal or very close to ambient (Fig. 6). The SO emission is elongated perpendicular to the $\mathrm{CO}$ outflow direction, which propagates across the SO region -limited by the contour at half-power- and finishes $\approx 40^{\prime \prime}$ Southeast of the SO peak. Both ambient SO and high-velocity CO brightness peaks are very close to each other (less than $10^{\prime \prime}$ ) and to knot HH 2L, where shocked molecular gas is detected (see e.g. Lefloch et al. 2003). This peak coincides with the maximum of density and of SO column density. Analysis of the physical conditions shows that the gas is denser than in the ambient parental cloud and could be warmer than the $30 \mathrm{~K}$ estimated from CO. The detection of high-velocity gas at the position where the density is highest suggests that SO is tracing the impact of the flow in the cloud. Also, the amount of SO material detected along the $\mathrm{HH} 2$ jet and the layers downstream of $\mathrm{HH} 2$ are similar (to less than a factor of 3 ), which suggests a common mechanism, and the column densities derived are typical of protostellar outflows.

Because the inclination of the flow with respect to the line of sight is very large, it is difficult to determine at which velocity SO emission occurs when the flow impacts the gas layers. Detailed modelling would help constrain the physical conditions of the shock that could account for the SO emission detected or if on the contrary UV radiation plays an important role in the emission detected.

\section{A cavity in the cloud}

In the previous section, we showed evidence that the protostellar outflow from VLA 1 has propagated beyond HH 2. Its impact on the ambient gas causes shocks which are detected in high-density tracers such as SO. Previous studies of the gas and dust structure in multiple star forming regions suggest that flows can have some other impact on the cloud dynamics; the study of NGC 1333 revealed for instance the presence of cavities in the cloud, apparently dug by the protostellar outflows (see e.g. Lefloch et al. 1998). We study in this section the largescale distribution of the molecular gas kinematics around $\mathrm{HH} 2$, to search for any similar impact of the outflowing gas on the parental cloud.

\subsection{The low-density gas around $\mathrm{HH} 2$}

We have studied the distribution of the low-density molecular gas by mapping the emission of the ${ }^{13} \mathrm{CO}(2-1)$ line. We present in Fig. 7 the map of the velocity-integrated flux in the range $5-11 \mathrm{~km} \mathrm{~s}^{-1}$. Three regions can be distinguished depending on the strength of the emission: the ambient cloud, between 8 and $9 \mathrm{~km} \mathrm{~s}^{-1}$; a fragment downstream of $\mathrm{HH} \mathrm{2}$, between 5 and $8 \mathrm{~km} \mathrm{~s}^{-1}$; the "filament" southwest of the protostellar core, between 10 and $11 \mathrm{~km} \mathrm{~s}^{-1}$. 


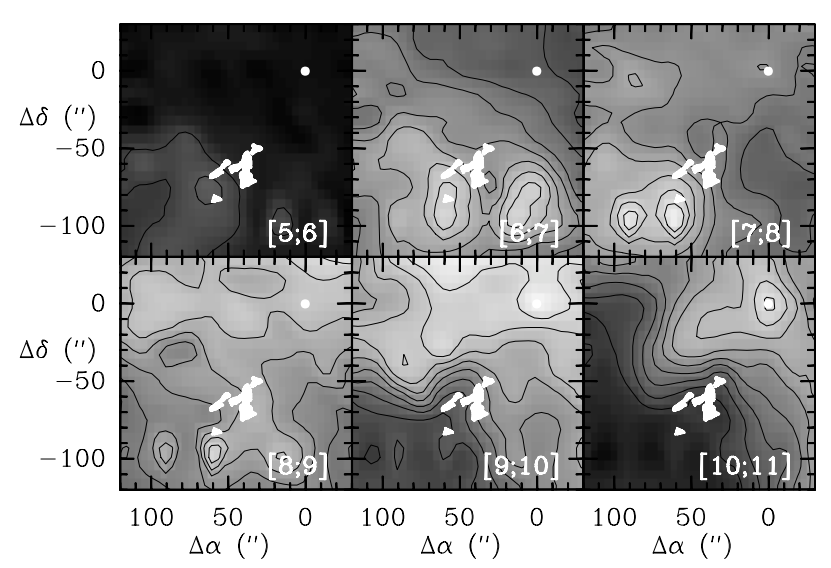

Fig. 7. Map of the velocity-integrated ${ }^{13} \mathrm{CO}(2-1)$ intensity in the $\mathrm{HH} 2$ region. First contour and contour interval are 2 and $1 \mathrm{~K} \mathrm{~km} \mathrm{~s}^{-1}$ respectively. Velocity interval is indicated in the bottom right corner. The invidual HH knots are drawn in white.

We do not find any evidence of a large-scale velocity gradient in the cloud. The velocity field in the cloud and the "filaments" are rather smooth. The latter overlaps very well with the border of the cloud. On the contrary, there is a striking morphological association between the layers at blue velocities $\left(5-8 \mathrm{~km} \mathrm{~s}^{-1}\right)$ and the ambient cloud (see right panel in Fig. 1): the integrated emission of the fragment matches exactly the border of the ambient cloud, which is possible only because there is barely any ambient emission.

We detect two local maxima in the fragment downstream of HH 2: one coincides with the "molecular emission peak" and the other peaks at the center of the optical "ring" at offset position $\left(15^{\prime \prime},-90^{\prime \prime}\right)$ (see Fig. 8d). The gas in the fragment peaks at $\approx 6.3 \mathrm{~km} \mathrm{~s}^{-1}$. The emission is characterized by bright lines $(\sim 10 \mathrm{~K})$ with a narrow linewidth $\left(\sim 1 \mathrm{~km} \mathrm{~s}^{-1}\right.$; see offset position (15", $\left.-75^{\prime \prime}\right)$ in Fig. 2).

An LVG calculation performed with a temperature of $30 \mathrm{~K}$ gives a column density $N\left(\mathrm{H}_{2}\right) \simeq 6.0 \times 10^{21} \mathrm{~cm}^{-2}$ at both positions in the layers. We note that the line opacity is quite high ( $\simeq 0.68)$; it is probably the reason for the lack of contrast in the emission. The gas column density derived is in rough agreement with the determination obtained by Dent et al. (2003), who found $N\left(\mathrm{H}_{2}\right)=2 \times 10^{22} \mathrm{~cm}^{-2}$ at the peak from dust millimeter continuum observations. From the mean $\mathrm{H}_{2}$ density in the gas $\left(\sim 2 \times 10^{5} \mathrm{~cm}^{-3}\right)$, we find that the thickness of the emitting region is $\sim 3-6 \times 10^{16} \mathrm{~cm}\left(5^{\prime \prime}-10^{\prime \prime}\right)$, much smaller than the extent over the sky. The emission appears to be distributed in a sheet of dense gas rather than in a "round clump". This means that the dense "ambient" gas emission is distribued around a cavity.

\subsection{Shocked $\mathrm{H}_{2}$ around the cavity}

Our ISOCAM/CVF observations (Fig. 8d) reveal some weak $\mathrm{H}_{2}$ emission in the $\mathrm{S}(2)$ and the $\mathrm{S}(3)$ lines above the $4 \sigma$ level in the gas shell where the second ${ }^{13} \mathrm{CO}$ maximum is detected - offset position $\left(15^{\prime \prime},-90^{\prime \prime}\right)$. The $\mathrm{H}_{2}$ emission is detected at the border of the gas layer, where the extinction is much less $\left(A_{\mathrm{v}} \simeq 4\right.$ at the molecular peak). The maximum of absorption in the center of the gas shell at $\left.\left(15^{\prime \prime},-90^{\prime \prime}\right)\right)$ is responsible for the annular appearance of the structure.

The $\mathrm{H}_{2}$ emission follows the distribution of the midinfrared continuum and the $11.3 \mu \mathrm{m}$ PAH band. The CVF spectra obtained towards the ring are similar to those of low-excitation galactic Photon Dominated Regions, such as Chamameleon, which are exposed to a low FUV field of a few ten times the ISRF (Boulanger et al. 1998). In none of the galatic PDRs of similar excitation conditions has $\mathrm{H}_{2}$ been detected. Therefore, we conclude that the $S(2)$ and $S(3)$ lines are tracing shocked gas over the shell. The non-detection of higher- $J \mathrm{H}_{2}$ transitions suggests low-excitation conditions, perhaps due to low-velocity shocks or previous (old) shocks. This strengthens our hypothesis that the shell of dense gas has been shaped by shocks, most likely from outflows.

It is therefore no wonder that the mid-IR emission map shows no correlation with the millimeter thermal dust emission, which traces the "cold" dust. This component is detected mainly in the protostellar condensation VLA 1-4 and in the layers ahead of HH 2 (Dent et al. 2003). The ring and the jet coincide with a minimum in the "cold" dust emission. Conversely, the maximum of absorption in the layer ahead of HH 2 prevents any mid-IR radiation from escaping the border of the cavity.

\subsection{Dust grain properties}

The dust grains in the ring exhibit properties very different from those observed in the protostellar envelopes of VLA 1-4. In the ring, we do not find any evidence at all of the presence of mantle ices. The silicate absorption is so large towards VLA 1 that it makes it difficult to analyse the mantle composition. The lower absorption in the envelope of VLA 4 allows us to characterize the composition of the mantle ices: $\mathrm{H}_{2} \mathrm{O}, \mathrm{CH}_{3} \mathrm{OH}, \mathrm{CH}_{4}, \mathrm{CO}_{2}$ (Cernicharo et al. 2000). A broad bump from 11 to about $14 \mu \mathrm{m}$ is detected all over the ring. Such a bump is interpreted as the signature of crystalline silicates, although the exact composition would require complementary data at longer wavelengths. Note that crystalline silicates have also been detected around the CS star (Cernicharo et al. 2000). Standard dust grains are expected to undergo deep changes in their composition when crossing shock(s) in the protostellar jet, as they release their icy mantle into the gas phase, either because of sputtering or shattering (Jones et al. 1994). Such processes result in an enrichment of the population of very small grains. Most likely, the very small grains detected by ISOCAM in the jet and in the ring are the result of this enrichment. In this context, a simple explanation for the detection of crystalline silicates is that they were originally present in grain cores and had already started to crystallize before the mantles evaporated. Further spectroscopy with the instruments onboard SPITZER should allow us to better constrain the composition and the physical parameters of the dust grains. As discussed above, the peculiar gas composition ahead of HH 2 most likely results from shock(s) too. Molecular line observations at higher angular resolution should be undertaken to better constrain the parameters of the shock. 
a)

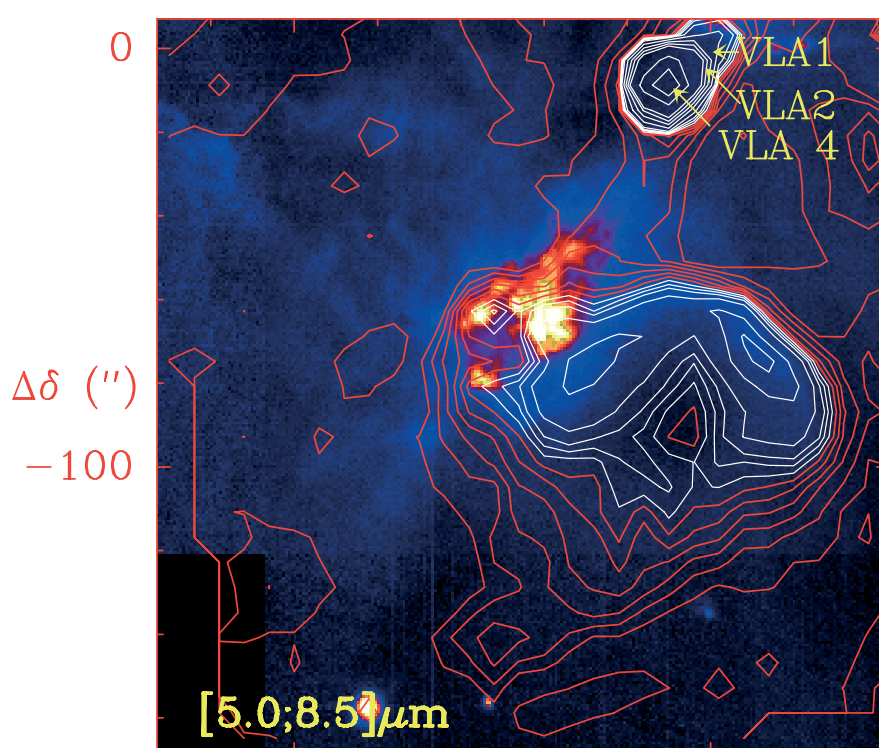

b)

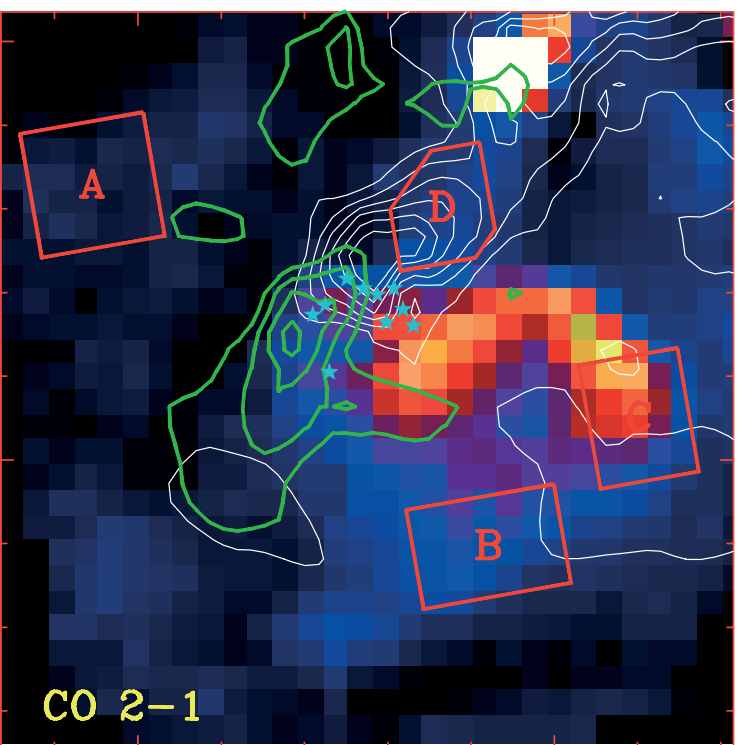

CO 2-1

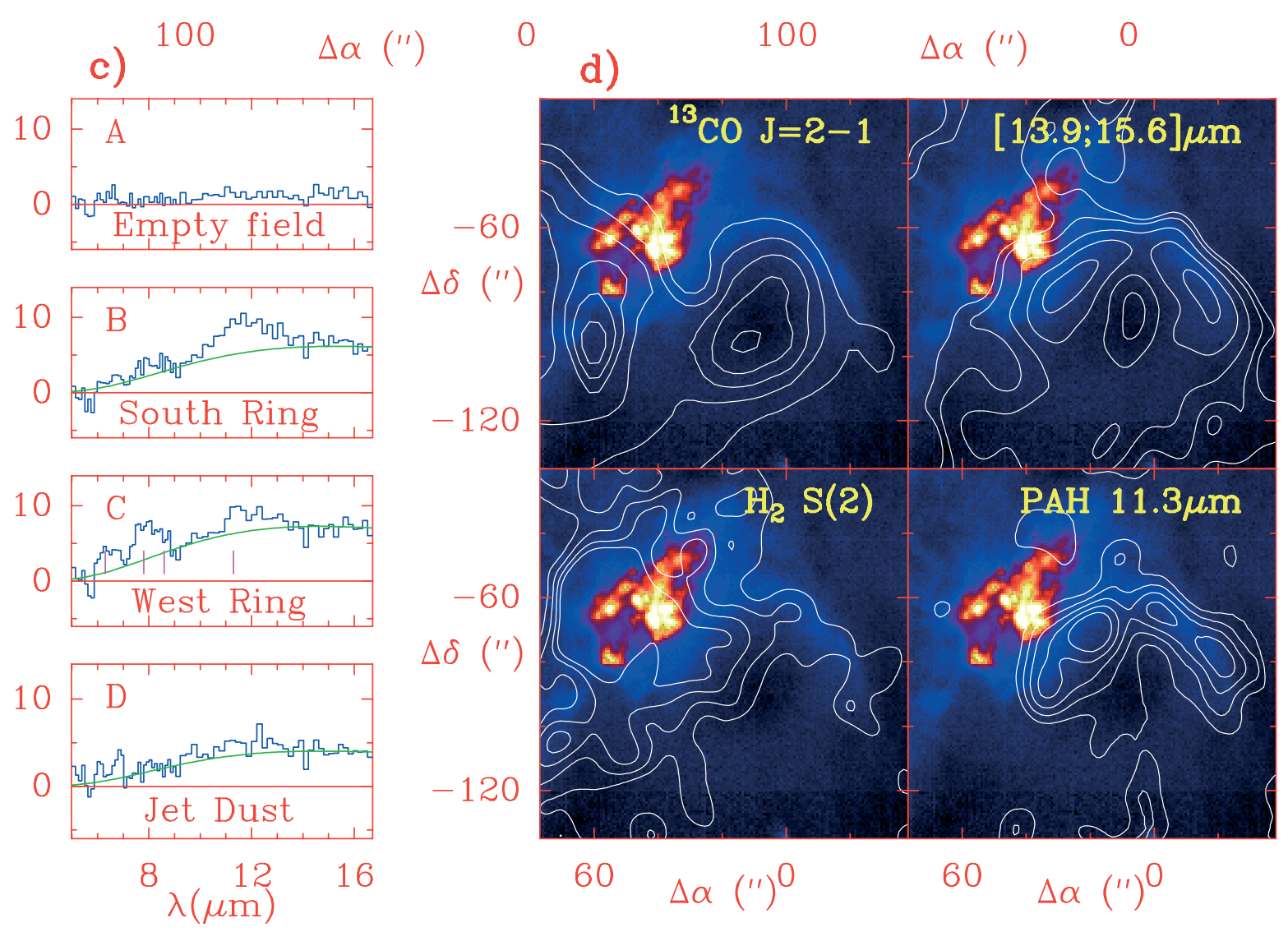

Fig. 8. a) Mid-infrared emission integrated between 5 and $8.5 \mu \mathrm{m}$ superposed on an optical [S 2] image of HH 2 (Reipurth 1993). Contours range from 3 to $5.6 \mathrm{mJy}$ by step of $0.2 \mathrm{mJy} / \mathrm{pixel}$ (px), from 6 to $10 \mathrm{mJy}$ by step of $1 \mathrm{mJy} / \mathrm{px}$, from 15 to $20 \mathrm{mJy}$ by step of $5 \mathrm{mJy} / \mathrm{px}$. b) Highvelocity $\mathrm{COJ}=2 \rightarrow 1$ emission (white contours) superposed on the 5.0-8.5 $\mu \mathrm{m}$ integrated mid-infrared map (colourscale). Contours range from 4 to $20 \mathrm{~K} \mathrm{~km} \mathrm{~s}^{-1}$ by step of $2 \mathrm{~K} \mathrm{~km} \mathrm{~s}^{-1}$. The position of the individual shocks A-L are marked with stars. Red polygons delineate the fields used to compute individual spectra. c) Spectra of the mid-infrared emission between 5.0 and $17 \mu \mathrm{m}$. d) Emission detected towards the "ring" West of $\mathrm{HH} 2 \mathrm{H}$ in: ${ }^{13} \mathrm{CO}(2-1)$ : contours range from 6 to $11 \mathrm{~K} \mathrm{~km} \mathrm{~s}^{-1}$ by step of $1 \mathrm{~K} \mathrm{~km} \mathrm{~s}^{-1} ; \mathrm{H}_{2} \mathrm{~S}(2) 12.2 \mu$ m: contours range from 4 to $10 \mathrm{mJy}$ by step of $2 \mathrm{mJy} / \mathrm{px} ; 13.9-15.4 \mu \mathrm{m}$ continuum: first contour is $4 \mathrm{mJy}$, following contours range from $4.5 \mathrm{mJy}$ to $7.5 \mathrm{mJy}$ by step of $1 \mathrm{mJy} / \mathrm{px}$; PAH $11.3 \mu \mathrm{m}$ band: contours range from 2 to $4.5 \mathrm{mJy}$ by step of $0.5 \mathrm{mJy} / \mathrm{px}$. The CVF maps were convolved with a Gaussian of 12 " HPFW to outline the extended emission. The rms in the $11.3 \mu \mathrm{m} \mathrm{PAH} \mathrm{map} \mathrm{and} \mathrm{the} \mathrm{continuum} \mathrm{map} \mathrm{is} 0.3 \mathrm{mJy} / \mathrm{px}$; it is $\approx 1 \mathrm{mJy} / \mathrm{px}$ in the $\mathrm{H}_{2} \mathrm{~S}(2) \mathrm{map}$. 


\subsection{What is the origin of the cavity?}

The peculiar geometry of the gas fragment downstream of HH 2 suggests that it could have been detached from the parental cloud. At least two observational facts suggest that the outflow activity could be responsible for detaching this fragment. First, the border of the ambient cloud coincides with the location of the knots HH 2A-K, where violent, dissociative shocks are detected. Second, the outflow wing downstream of HH 2 coincides very well with the gas fragment, in the plane of the sky. Both gas components (the fragment and the outflow wing) are blueshifted with respect to the ambient cloud.

On the other hand, the momentum carried away by the blueshifted outflow wind is $\approx 0.1 M_{\odot} \mathrm{km} \mathrm{s}^{-1}$, i.e. a factor of a few less than the momentum necessary to detach the fragment from the cloud. The sum of several ejections in the past could have provided enough momentum; we speculate that another outflow, much more powerful, could be responsible for digging the cavity in the ambient cloud. When looking at the velocity channel map of SO emission (Fig. 3), it is interesting to note that the filament southwest of the core: a) consists of dense gas (SO); b) is velocity-shifted with respect to the ambient cloud; c) closely follows the border of the latter. Such a configuration is suggestive of a shock front propagating from the border of the cavity into the cloud. The orientation of such an outflow would probably be closer to North-South than the actual HH 1-2 jet.

\section{Photon-Dominated Region in $\mathrm{HH} 2$}

The emission integrated between 5 and $17 \mu \mathrm{m}$ is shown in Figs. $8 \mathrm{a}, \mathrm{b}$. The SNR rms is $\sim 0.1 \mathrm{mJy}$ in the map. Protostar VLA 4 (Cernicharo et al. 2000) is detected as a bright point source in Figs. 8a, b. After subtracting the contribution of the ambient cloud, estimated from a reference position, the residual flux appears very weak; a spectrum of the emission averaged over a reference field centered at $\left(+100^{\prime \prime},-35^{\prime \prime}\right)$ shows a contribution less than $\sim 1$ mJy (panel A in Fig. $8 \mathrm{c}$ ). Hence, the emission discussed here arises from the $\mathrm{HH} 2$ region. Southeast of the protostellar core down to $\mathrm{HH} 2$, we detect mid-infrared emission along the HH 2 jet. The average flux level was estimated over field D and is about $5 \mathrm{mJy}$ longwards of $10 \mu \mathrm{m}$ ( $5 \sigma$ above the rms noise level). The spatial coincidence with the CO high-velocity outflow (Fig. 1) suggests a physical assocation with the latter. The nature of the CVF emission appears to be mainly continuum in the jet, as indicated by the spectrum of Field D (Fig. 8c). This is the first time that mid-infrared continuum emission is reported towards a protostellar jet.

The map of the pure continuum between 13.9 and $15.4 \mu \mathrm{m}$ shows that most of the flux comes from a ring-like structure (hereafter the "ring") of $60^{\prime \prime}$ diameter $(0.13 \mathrm{pc})$, westwards of HH 2H (Fig. 8d), This annular structure is detected in the optical SII line (Reipurth et al. 1993) and in the 5.0-8.5 $\mu \mathrm{m}$ emission map. The minimum of emission in the center of the ring coincides with the peak of gas column density detected in ${ }^{13} \mathrm{CO}$, at offset position $\left(15^{\prime \prime},-75^{\prime \prime}\right)$. This provides direct evidence that the mid-IR "ring" and the molecular gas surrounding the cavity are closely related. The average spectrum of the ring obtained in field $\mathrm{C}$ shows the emission of the UIBs between 6.2 and $11.3 \mu \mathrm{m}$, which are a direct tracer of the local UV field. Identification is more difficult in the Southern part of the ring (field B); the SNR is not high enough to conclude about a gradient in the PAH abundance. However, the continuum emission detected in the South testifies to the fact that the whole gas layer is illuminated by UV photons, on the rear side.

The emitting region, as traced by the $11.3 \mu \mathrm{m}$ map (Fig. 8d), coincides very well with the pure continuum emission distribution. In the jet, on the contrary, we note that there is no hint of PAH emission bands. The relatively low SNR and the strong cloud extinction prevent us from drawing a conclusion about the presence/absence of PAHs along the jet.

A rough estimate of the FUV field intensity is obtained by integrating the flux in the ISOCAM band. This yields an infrared luminosity $L \sim 0.01 L_{\odot}$, and a FUV field: $G=20 \mathrm{G}_{0}$. This is fully consistent with the estimate obtained by Molinari \& Noriega-Crespo (2002) from the analysis of the FIR [CII] $158 \mu \mathrm{m}$ and [OI] $63 \mu \mathrm{m}, 145 \mu \mathrm{m}$ lines and the FIR continuum observed at $80^{\prime \prime}$ resolution with ISO/LWS. We note that our direct estimate of the FUV lies in the low range of values $\left(20-1000 \mathrm{G}_{0}\right)$ required by the models of UV-driven photochemistry (Viti et al. 2003) to account for the molecular emission ahead of HH 2.

Continuum emission in the jet and the ring could be fitted by a greybody with an opacity law $\tau_{v} \propto v$ and a temperature $T_{\mathrm{d}}=200-250 \mathrm{~K}$. Assuming that the emission comes from typical interstellar a(big) dust grains, we find that the amount of material corresponds to an $A_{\mathrm{v}} \simeq 0.1-1 \times 10^{-6}$, which translates into very low gas column densities. The efficiency of dust grains with a size as small as $0.003 \mu \mathrm{m}$ is too low (Draine \& Lee 1984) to reach equilibrium temperatures of this order, unless the UV field is actually very strong, far above our estimate. Most likely, we are observing the emission of very small dust grains, transiently heated to high temperatures by the local UV field.

Weak continuum flux is also detected across the HH object and West of the protostellar core VLA 1-4. This extension coincides with the border of the cavity in the molecular cloud.

\section{1. $\mathrm{HH} 2 \mathrm{H}$ as exciting source of the PDR}

Previous observations in the UV have revealed strong extended emission in the direction of $\mathrm{HH} 2 \mathrm{H}-2 \mathrm{~A}$ (Böhm-Vitense et al. 1982; Raymond et al. 1997). The other sources in the field detected by IUE are HH 1 and the Cohen-Schwartz star; both are located too far away to account for the PDR observed around HH 2. Analysis of optical high-ionization lines has shown evidence for a $J$-type shock with velocities of $\sim 180 \mathrm{~km} \mathrm{~s}^{-1}$ moving into previously accelerated gas (optical measurements indicate proper motions up to $400 \mathrm{~km} \mathrm{~s}^{-1}$ towards $\mathrm{HH} 2 \mathrm{H}$ and $\mathrm{HH} 2 \mathrm{~A}$ (Bally et al. 2002), identifying $\mathrm{HH} 2 \mathrm{H}$ as the actual jet impact region (or Mach disk region). $\mathrm{HH} 2 \mathrm{H}-2 \mathrm{~A}$ is therefore the most plausible candidate as an exciting source; it is also the only place where X-ray emission has been detected in the region (Pravdo 2001). 
HUT measurements by Raymond et al. (1997) show that the UV continuum is dominated by 2 photon radiation of collisionally excited $\mathrm{H}$ atoms longwards of $1750 \AA$. At shorter wavelengths, the continuum is dominated by $\mathrm{H}_{2}$ Lyman emission bands. Once corrected for the interstellar extinction, the total UV luminosity is $\sim 0.3 L_{\odot}$; it is distributed roughly equally between both mechanisms. The exact solid angle encompassed by the ring is difficult to estimate. Following our hypothesis that the outflow has dug a cavity around $\mathrm{HH} 2$, it is reasonable to assume that the ring and the molecular emission peak ahead of $\mathrm{HH} 2$ are at about the same distance to the UV source, knots H-A $\left(50^{\prime \prime}\right)$. The diameter of the ring is $60^{\prime \prime}$, hence the fraction of photons intercepted by the ring is $\sim 5 \%$, which compares well with the mid-IR luminosity radiated by the very small grains in the walls of the cavity ( $3 \%$ of the UV luminosity produced in $\mathrm{HH} 2 \mathrm{H}-2 \mathrm{~A}$ ).

The possibility of a similar mechanism (strong $J$-shocks) to account for the mid-infrared emission along the protostellar jet, between VLA 1 and HH 2, should be explored. Deep sensitive spectroscopy of the entrained gas could allow one to constrain such possibility. Another possibility is that the jet region is actually almost free of dust grains after the crossing of the strong shocks which are now impacting HH 2. In this case we would be detecting the walls of a dust-free cavity around the jet, illuminated either by $\mathrm{HH} 2 \mathrm{H}$ or by the powering source.

\section{Summary and conclusions}

The observations presented in this paper allow us to draw a more complete and complex picture of the HH 2 region. In addition to the high-velocity molecular outflow associated with the optical jet HH 1-2, we have detected another outflow component that propagates over $50^{\prime \prime}$ in the molecular gas downstream HH 2. This outflow is associated with a weak jet detected in the optical, which appears to come from HH 2. The orientation of this component in the sky differs from the $\mathrm{HH} 2$ jet and could be the result of jet deflection on the dense obstacles responsible for the knots of $\mathrm{H}_{2}$ emission in $\mathrm{HH} 2$. Another possibility is that this component traces a previous ejection from the source, which would be precessing. High angular resolution observations should allow us to test the first hypothesis, by comparison with numerical modelling (see e.g. Raga \& Canto 1995)

This provides direct evidence that the molecular gas ahead of HH 2 has been affected by protostellar ejections. It is consistent with the detection of optical bow-shocks at large distances from the HH 1-2 region. Several kinematical components are detected in the gas downstream of $\mathrm{HH} 2$. Observations of SO sreveal the presence of very dense gas $\left(\sim 2-5 \times 10^{5} \mathrm{~cm}^{-3}\right)$ associated with the low-velocity shocks along the $\mathrm{HH} 2$ jet, and in the blueshifted outflow, close to knot HH 2L. The latter position coincides with the peak of emission in the region. We conclude that SO is probably tracing the shock interaction of the outflow with the ambient gas.

The gas layers downstream of HH 2 exhibit evidence of shocks: East of $\mathrm{HH} 2$, ISOCAM observations detect $\mathrm{H}_{2}$ line emission in the gas layers, which is the signature of shocks, probably old enough to be detected only in the low-excitation transitions $\mathrm{S}(2)$ and $\mathrm{S}(3)$. The gas layers have been shaped in a shell, probably as the result of prostellar outflow interaction with the cloud. Actually, the morphology and kinematics of the filament Southwest of the protostellar core are compatible with their tracing a shock-compressed "back side" of the cavity (the "front side" being the gas shell downstream of $\mathrm{HH}$ 2).

ISOCAM observations show emission from very small grains at the inner surface of the cavity downstream of $\mathrm{HH} 2$. This could result from the shattering and sputtering of large interstellar dust grains in the outflow/cloud interaction. We find that the UV field produced in the strong shock $\mathrm{HH} 2 \mathrm{H}-\mathrm{A}$, which illuminates the inner side of the cavity, creates a PhotonDominated Region of FUV intensity $G \sim 20 \mathrm{G}_{0}$. It is sufficient to account for the emission of the very small grains and the PAHs detected. Mid-infrared emission along the jet remains a puzzle; deep mid-infrared spectroscopy along the jet could allow one to determine the origin of the emission, in particular if it is related to strong dissociative shocks between the source and $\mathrm{HH} 2$.

Our observations indicate that some of the molecular species whose abundance is found enhanced in the gas downstream of $\mathrm{HH} 2$ are actually produced in the region of outflow interaction with the cloud. Mapping at better angular resolution, comparable to the data presented here, should be undertaken. In combination with detailed shock modelling, it would allow us to explore the shock hypothesis and estimate its relative contribution with respect to UV-induced photodesorption in the gas phase enrichment of "unusual" molecular species.

Acknowledgements. J. Cernicharo acknowledges the Spanish DGES for this research under grants AYA2000-1784 and AYA2003-2784. We thank Dr. F. Boulanger for many stimulating discussions on the ISOCAM observations of $\mathrm{HH} 2$.

\section{References}

Bachiller, R., \& Perez Gutierrez, M. 1997, ApJ, 487, L93

Bally, J., Heathcote, S., Reipurth, B., et al. 2002, ApJ, 123, 2667

Böhm-Vitense, E., Böhm, K. H., Cardell, J. A., \& Nemec, J. M. 1982, ApJ, 262, 224

Boulanger, F., Abergel, A., Bernard, J. P., et al. 1998, in Star Formation with the Infrared Space Observatory, ed. J. Yun, \& R. Liseau ASP Conf. Ser., 132, 15,

Cernicharo, J., Noriega-Crespo, A., Cesarsky, D., et al. 2000, Science, 288,649

Cesarsky, C. J., Abergel, A., Agnese, P., et al. 1996, A\&A, 315, L32

Davis, C. J., Dent, W. R. F., \& Bell Burnell, S. J. 1990, MNRAS, 244, 173

Dent, W. R. F., Furuya, R. S., \& Davis, C. J. 2003, 339, 633

Draine, B. T., \& Lee, H. M. 1984, ApJ, 285, 89

Fuente, A., Cernicharo, J., Barcia, A., \& Gomez-Gonzalez, J. 1990, A\&A, 231, 151

Girart, J. M., Viti, S., Williams, D. A., Estalella, R., \& Ho, P. T. P. 2002, A\&A, 388, 1004

Green, S. 1994, ApJ, 434, 188

Green, S., \& Chapman, S. 1978, ApJS, 37, 169

Hartmann, L., \& Raymond, J. C. 1984, ApJ, 276, 560 
Henney, W. J., Raga, A. C., \& Axon, D. J. 1994, ApJ, 427, 305

Hester, J. J., Stapelfeldt, K. R., \& Scowen, P. A. 1998, ApJ, 116, 372 Hollenbach, D., \& McKee, C. F. 1989, ApJ, 342, 306

Jones, A. P., Tielens, A. G. G. M., Hollenbach, D. J., \& McKee, C. F. 1994, ApJ, 433, 797

Kessler, M. F., Steinz, J. A., \& Anderegg, M. E. 1996, A\&A, 315, L27 Lefloch, B., Cernicharo, J., Cabrit, S., et al. 2003, ApJ, 590, L41

Martin-Pintado, J., \& Cernicharo, J. 1987, A\&A, 176, L27

Molinari, S., \& Noriega-Crespo, A. 2002, ApJ, 123, 2010

Moro-Martín, Cernicharo, J., Noriega-Crespo, A., \& Martín-Pintado, J. 1999, ApJ, 520, L111 (MM99)

Ogura, K. 1995, ApJ, 450, L23
Pravdo, S. H., Feigelson, E. D., Garmire, G., et al. 2001, Nature, 413, 708

Raga, A. C., \& Cantó, J. 1995, RmxAA, 31, 51

Rieke, G. H., \& Lebofsky, M. J. 1985, ApJ, 288, 618

Raymond, J., Blair, W. P., \& Long, K. S. 1997, ApJ, 489, 314

Reipurth, B., Heathcote, S., Roth, M., Noriega-Crespo, A., \& Raga, A. C. 1993, ApJ, 408, L408

Torelles, J. M., Rodriguez, L. F., Canto, J., et al. 1992, ApJ, 396, 95

Viti, S., \& Williams, D. A. 1999, MNRAS, 310, 517

Viti, S., Girart, J. M., Garrod, R., et al. 2003, A\&A, 399, 187

Wolfire, M. G., \& Königl, A. 1993, ApJ, 415, 204 